\title{
In-situ probe of the response of the Tile Calorimeter to isolated hadrons
}

\author{
David Jennens \\ On behalf of the ATLAS Collaboration
}




\section{The ATLAS Experiment}

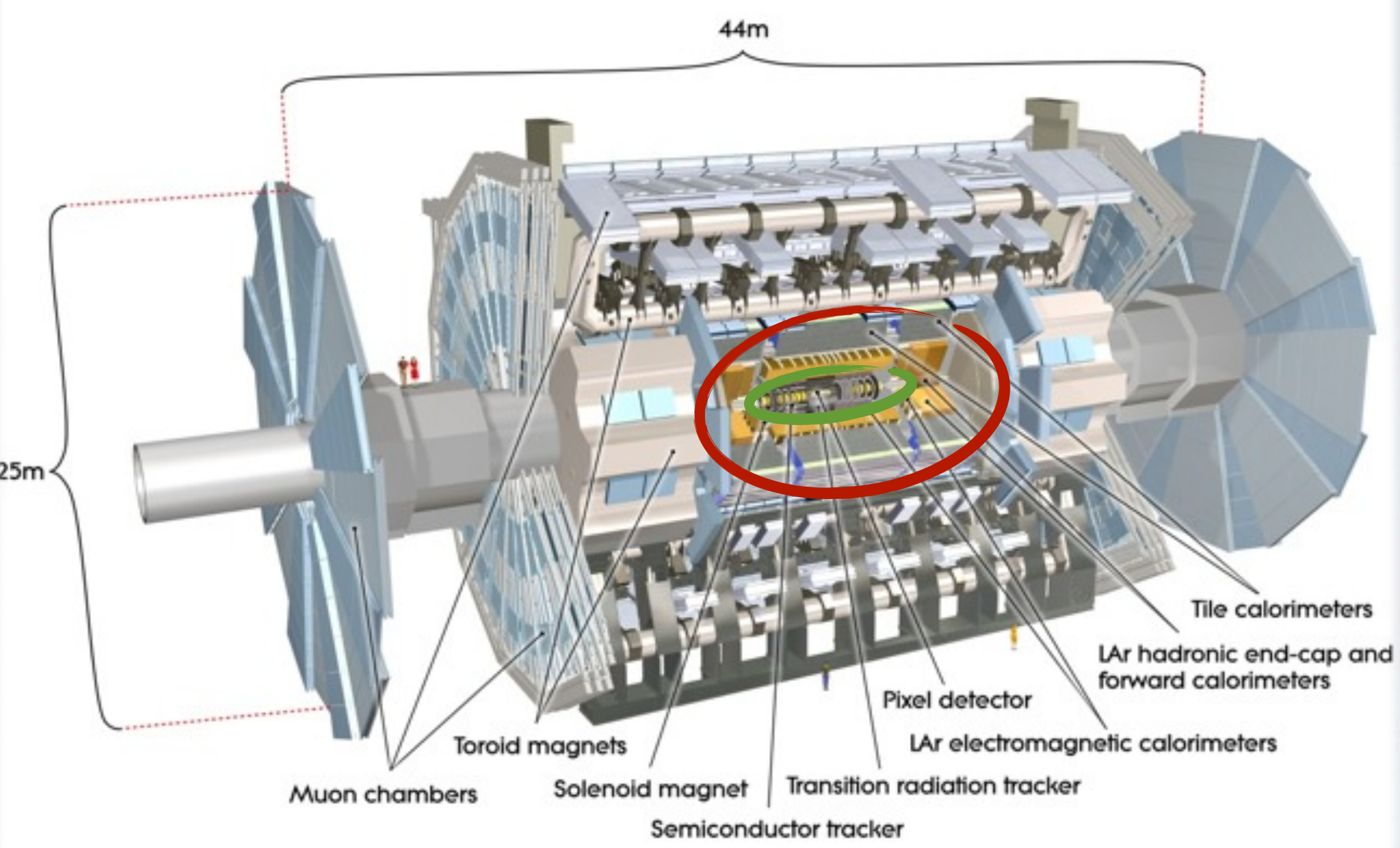

Comprised of:

Semiconductor tracker

I. The Inner Detector to measure the momentum of charged particles

2. The Calorimeters to measure the energy of electromagnetic and hadronic showers through the Liquid Argon and Tile calorimeters.

3. The Muon Chambers to measure the momentum of muons

In-situ probe of Tile Calorimeter response 


\section{The Tile Calorimeter}

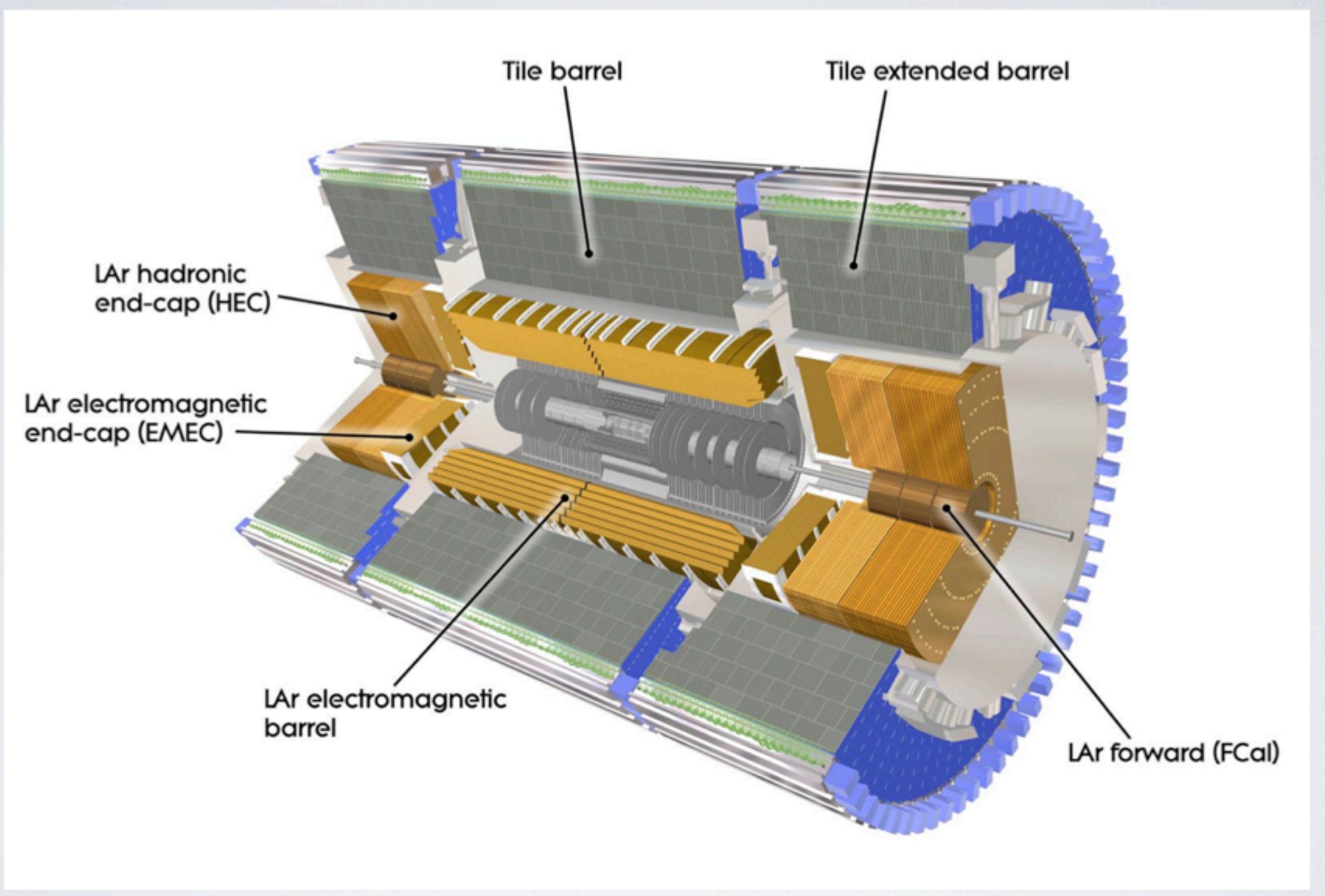

In-situ probe of Tile Calorimeter response 


\section{The Tile Calorimeter}

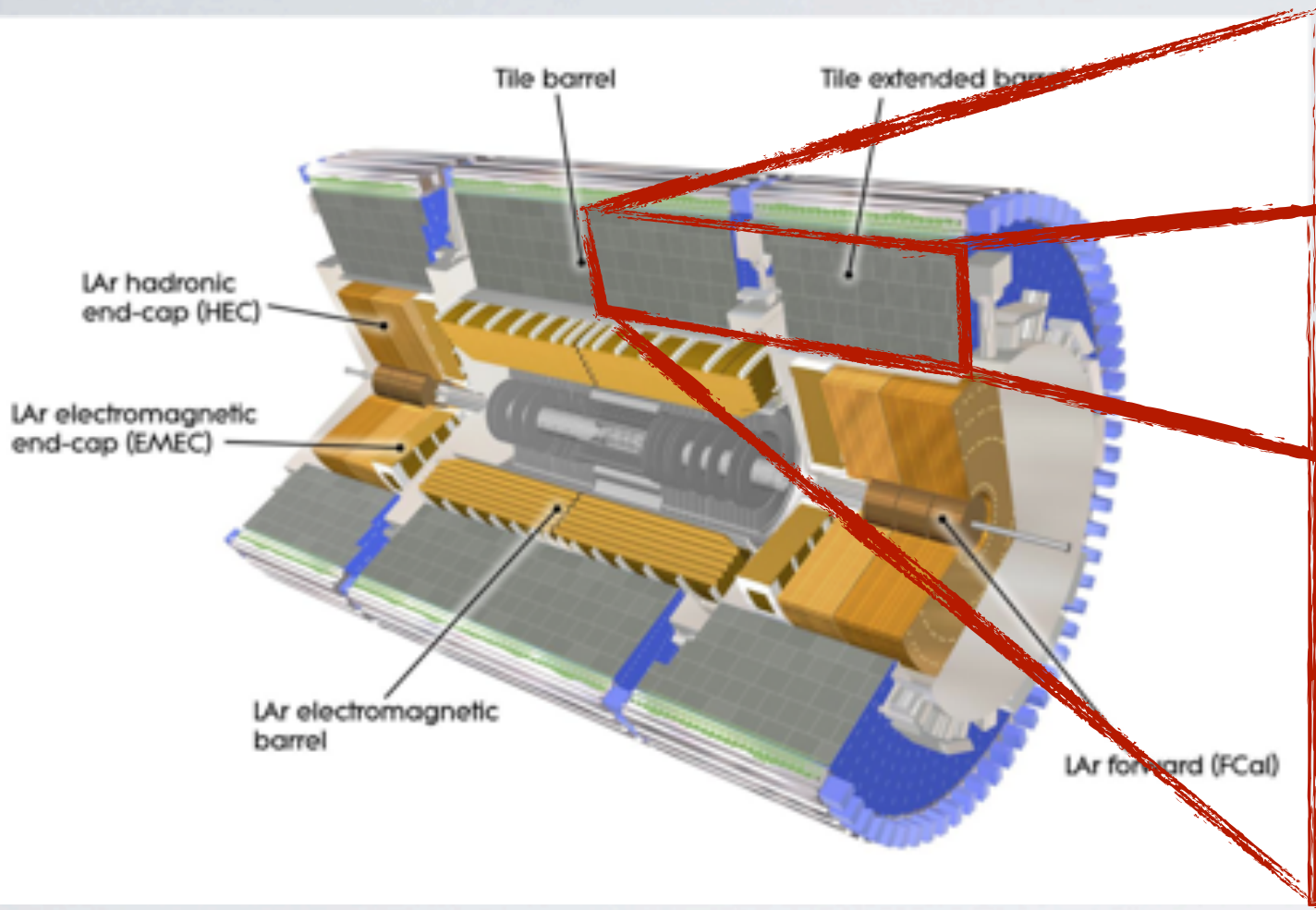

- Tile Calorimeter is divided into one long barrel $(|\eta|<0.7)$ and two extended barrels $(0.7<|\eta|<1.7)$

- Symmetric about the beam line in 64 modules in the $\phi$ direction

- Each module is segmented into 3 layers, A, BC and D. These layers are $1.5,4.1$ and 1.8 interaction lengths thick at $\eta=0$

- Layers are composed of cells with granularity $\Delta \eta \times \Delta \phi=0.1 \times 0.1$ or $0.1 \times 0.2$ for layer $D$.

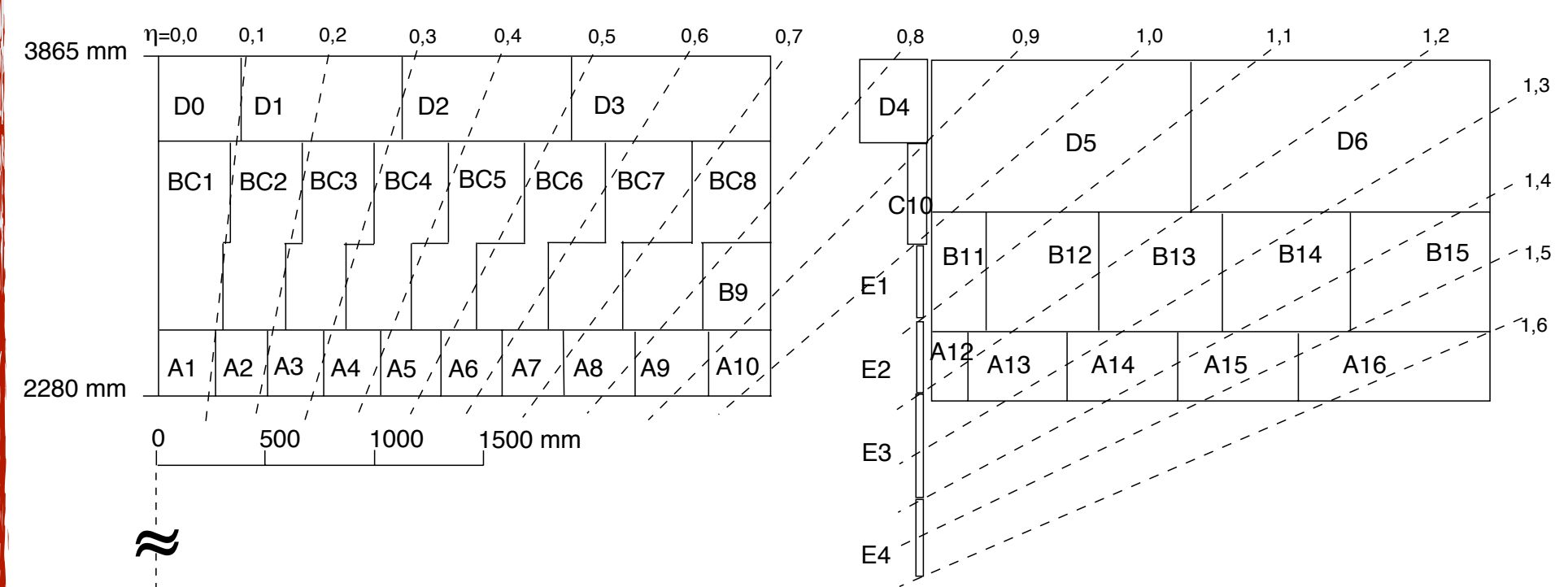

beam axis

$\rightarrow$

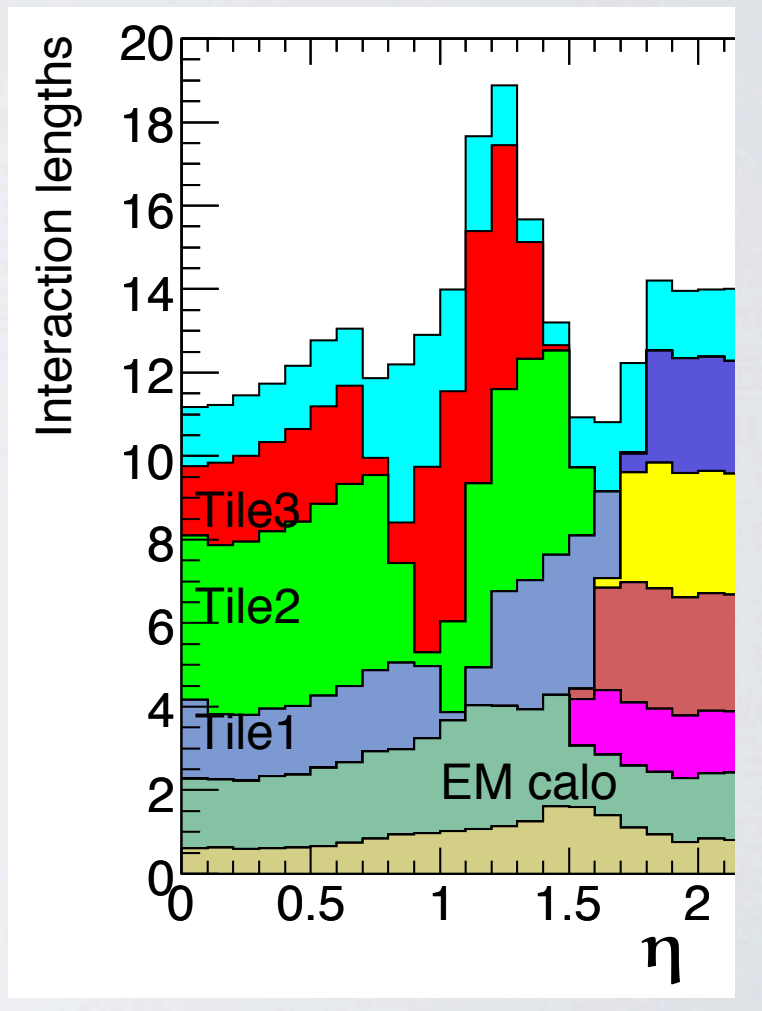

In-situ probe of Tile Calorimeter response 


\section{Calorimeter Response}

- The performance of the Tile Calorimeter system can be probed in-situ, via the response

- Measured Energy in Tile Calorimeter (E) vs True Energy

- For charged particles, the momentum ( $p$ ) observed using the Inner Detector can be used as a measure of the True Energy

- Inner Detector momentum resolution 1\% for the momentum range considered

- By studying the ratio E/P in both simulation and data the integrity of the simulation may be examined

Particle

Momentum ( $p)$

- $<E / p>$ is examined in bins of $\eta, p$ and $\phi$

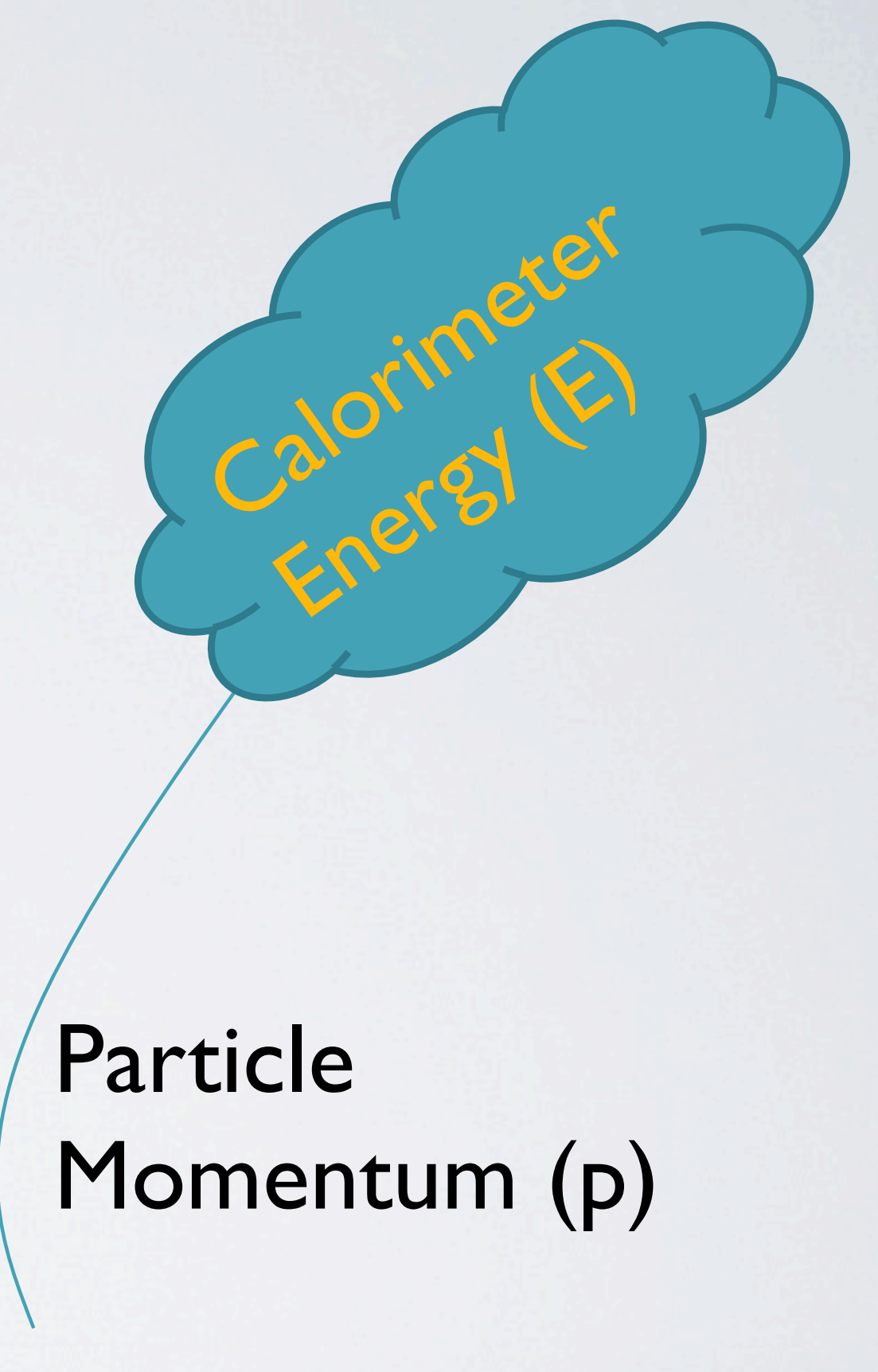

In-situ probe of Tile Calorimeter response 


\section{Methodology}

- Find charged hadrons (usually $\pi^{ \pm}$)

- Extrapolate their trajectory to each layer of the calorimeter system, using the known geometry of the detector and magnetic field strength.

- To remove contributions to the response from other particles, select particles isolated within a cone of $\Delta R=0.4$ from any inner detector track.

- Sum all the energy deposited in a cone around the charged hadron

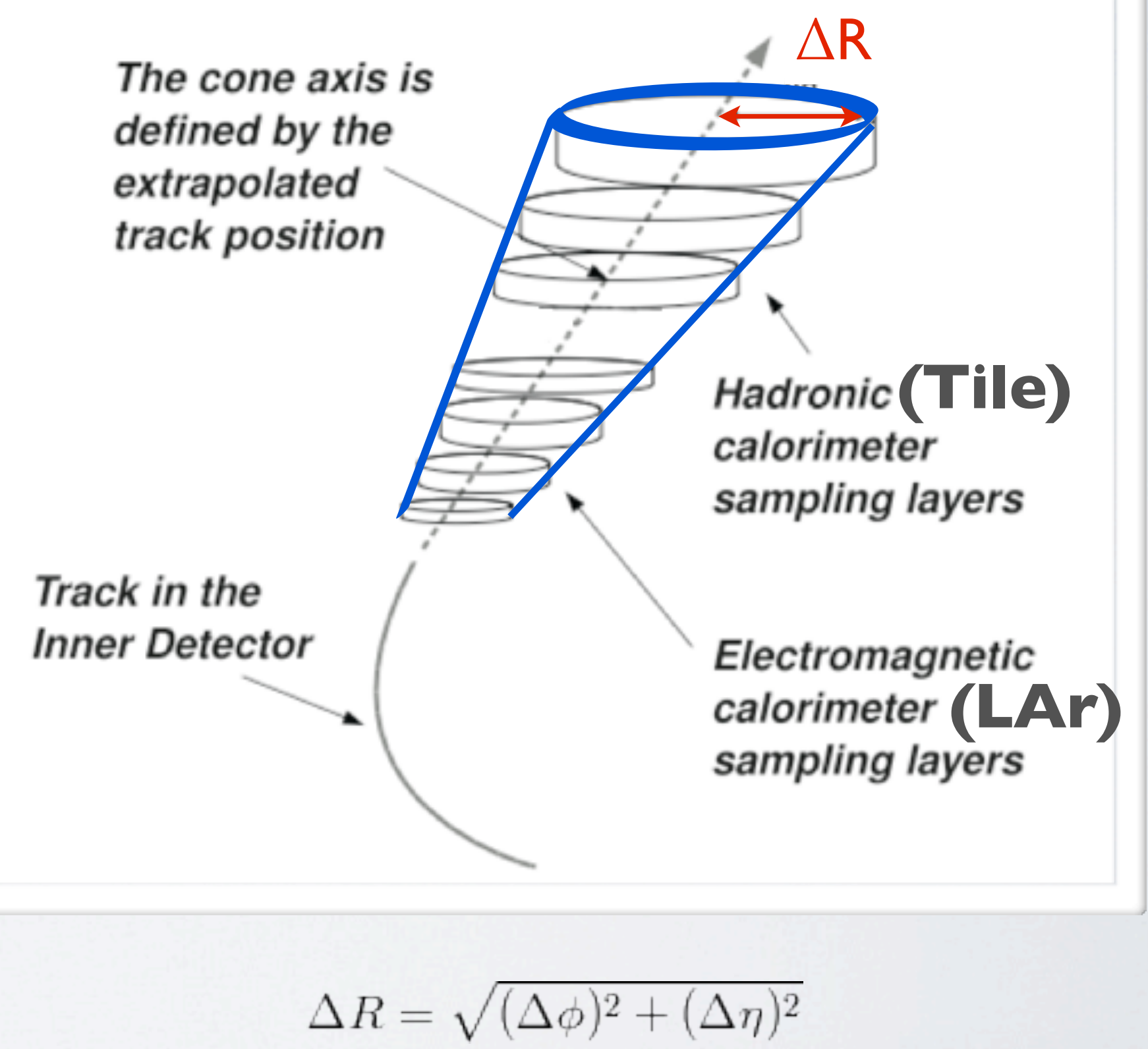

In-situ probe of Tile Calorimeter response 


\section{Choice of Cone Size}

- The best choice of cone size is found by comparing the response in:

- Single $\pi^{ \pm}$simulated sample (The response to a single particle)

\section{- Particles in a simulated minimum bias collision} (Background sources affect the energy around the isolated hadron)

- The choice is the best compromise between:

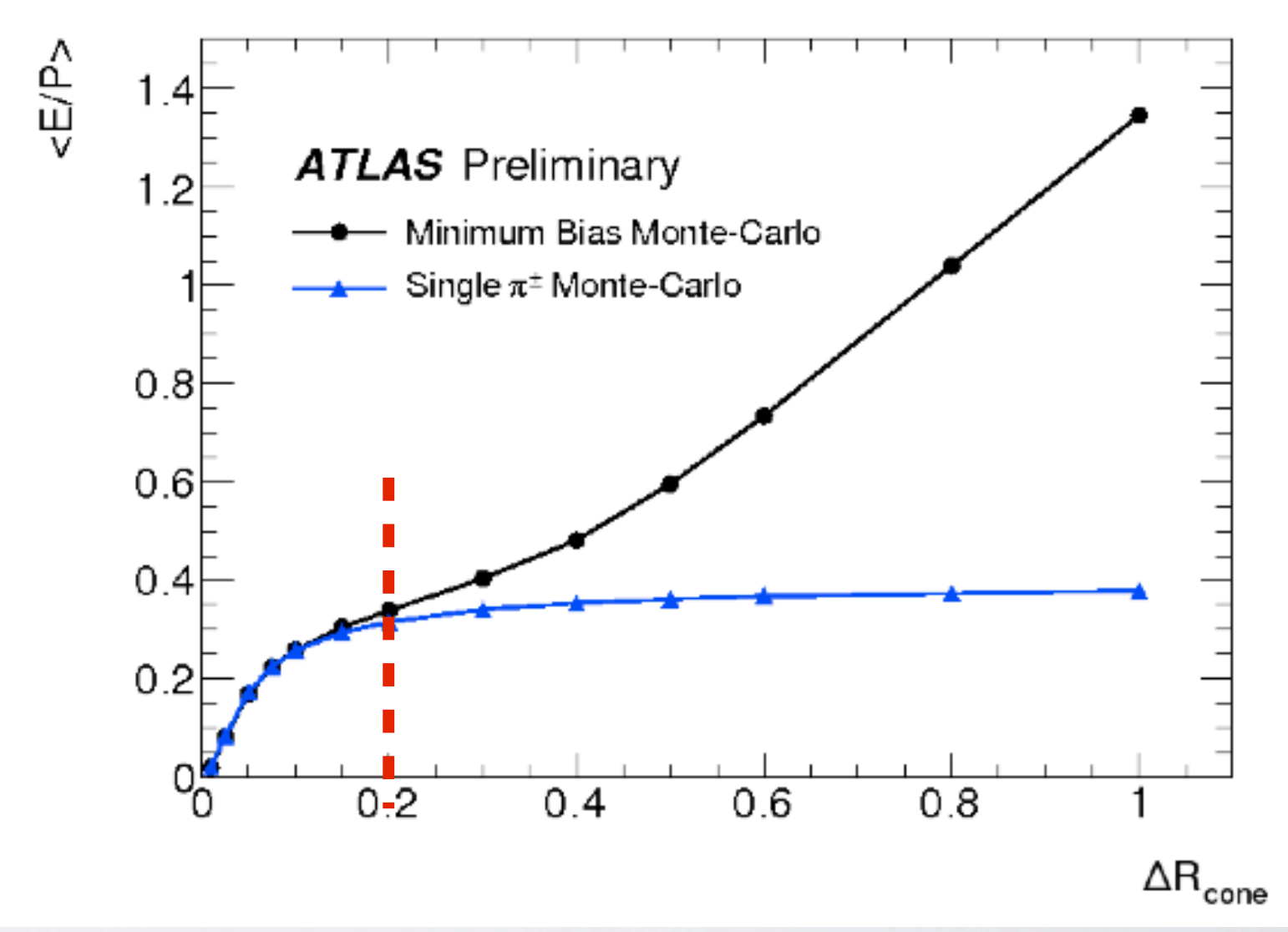

- Capturing all the energy associated with the charged hadron

- The $<E / p>$ of the single $\pi^{ \pm}$is close to it's maximum

- Avoiding energy from background sources in the event

- Minimising the difference between the minimum bias and the single $\pi^{ \pm}$radial energy deposition $\Delta R<0.2$ is chosen to best fit these constraints

In-situ probe of Tile Calorimeter response 


\section{Datasets Description}

- 2010 data events are triggered using the MBTS (Minimum Bias Trigger Scintillator).

- As the rate of minimum bias events is too high, approximately I in 500 events are triggered leaving $92 \mathrm{nb}^{-1}$.

- In 2010 bunch spacing was 150 ns and the average number of interactions per crossing was between 2 and 3 .

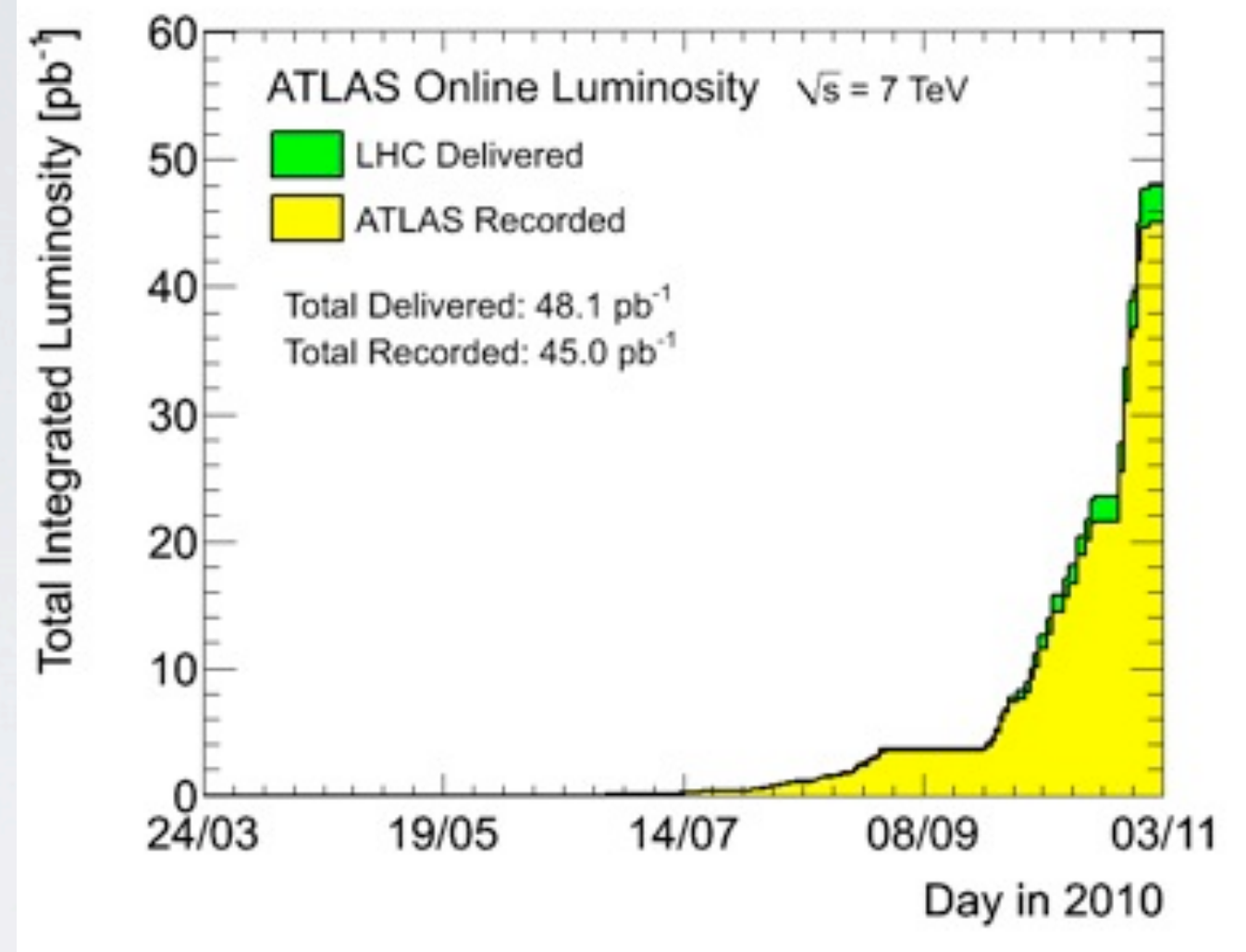

- 2011 data events are randomly triggered during collisions at a fixed rate.

- $4.7 \mathrm{fb}^{-1}$ is used from this year. The last period is not included.

- In 201 I bunch spacing was 50 or 75 ns and the average number of interactions per crossing was significantly higher, between 10 and 18.

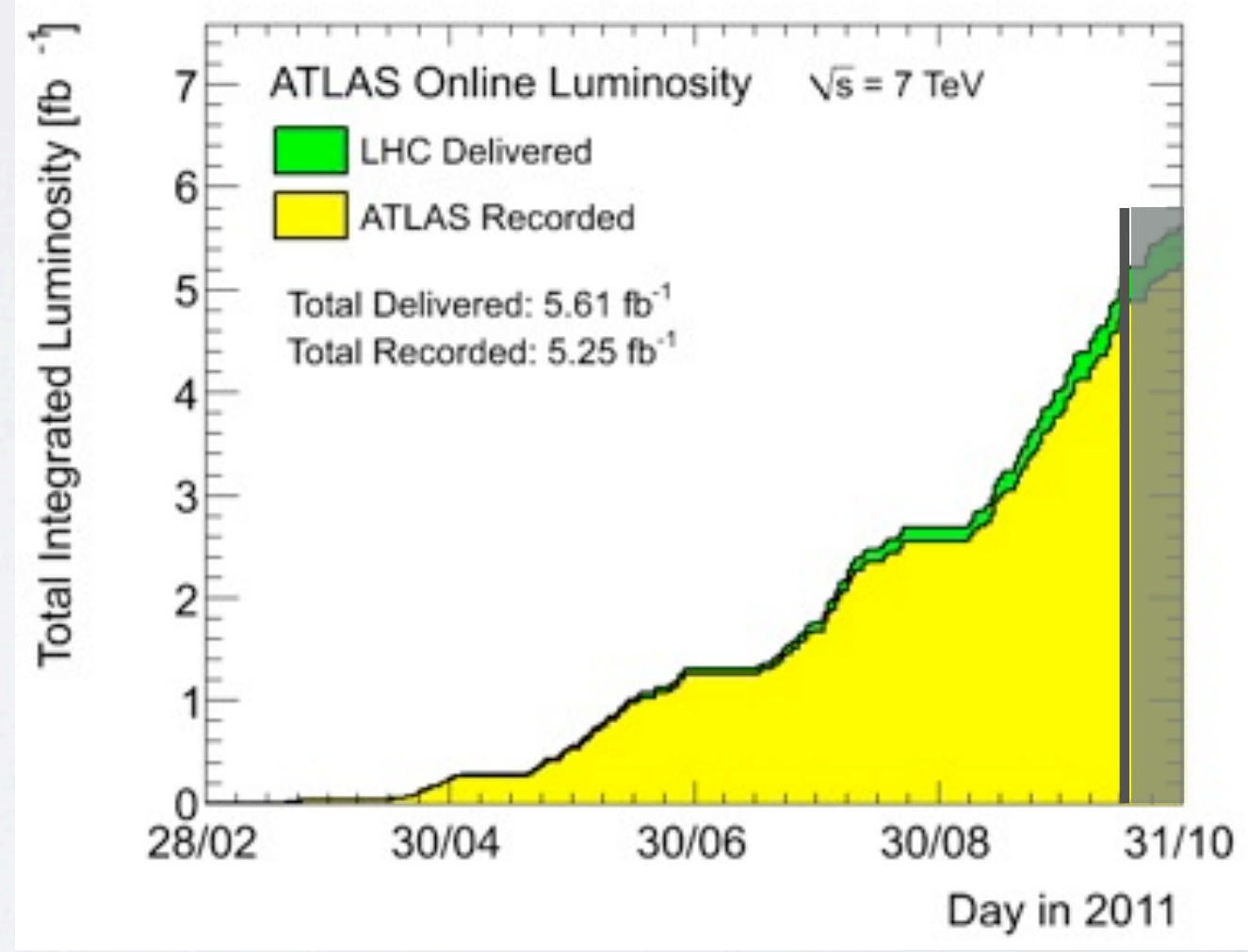

\section{In-situ probe of Tile Calorimeter response}




\section{Datasets Description}

- The simulated datasets for both 2010 and 2011 contain 20 million minimum bias events, generated using Pythia 6 .

- The hadronic interactions were simulated with the QGSP_BERT set of processes in Geant4

- The simulated sample is weighted so that it's pile-up distribution matches that observed in data

- Simulated events are normalised to observed events by deriving weights in bins of pseudo-rapidity and momentum (2010) or transverse momentum (20II).

- This normalisation does not affect the shape of the E/P distribution.
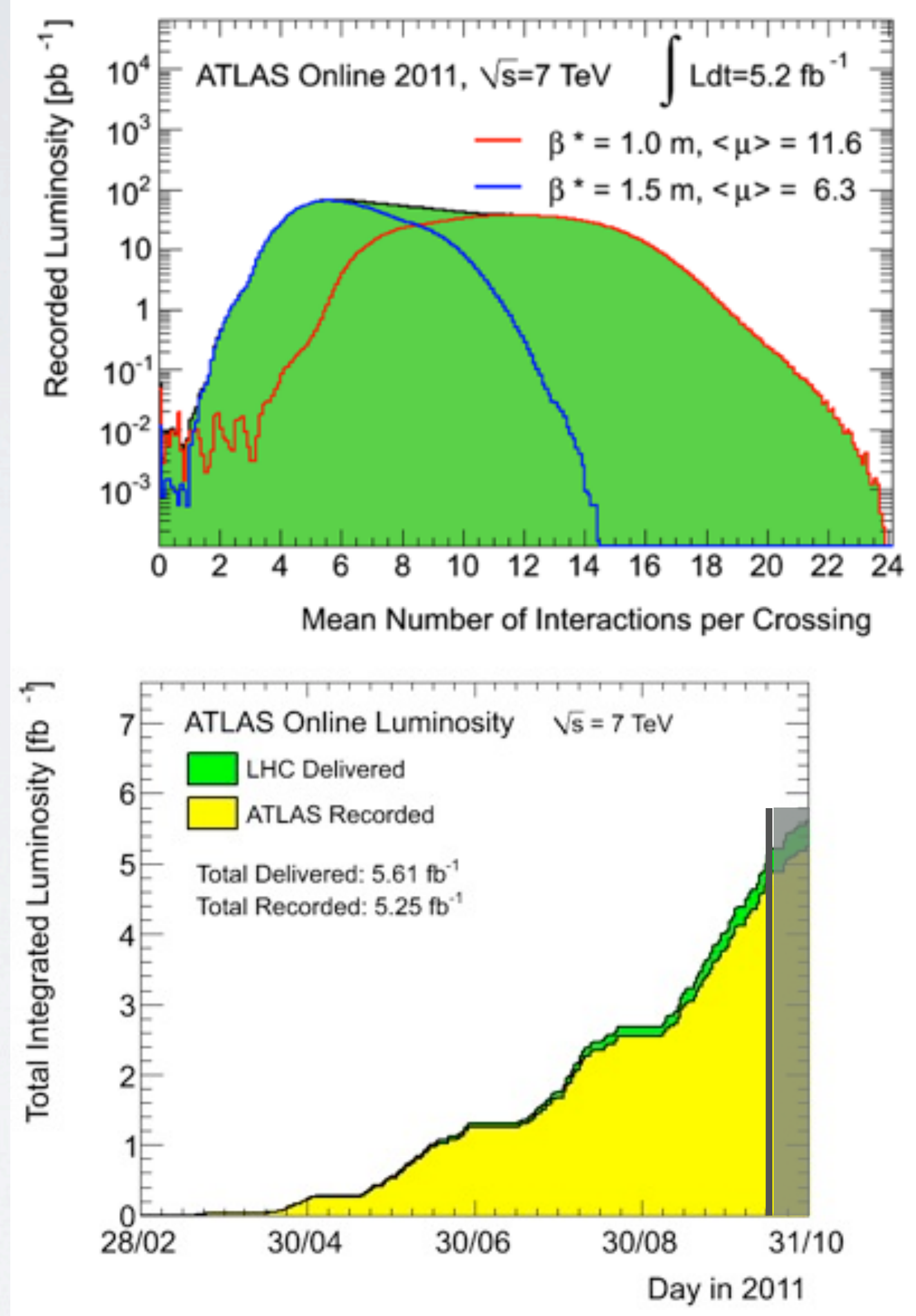

In-situ probe of Tile Calorimeter response 


\section{Particle Selection}

- Particles are selected to probe our understanding of Tile Calorimeter

- Particles are selected if no energy deposit associated with them comes from a calorimeter cell which has been flagged as problematic

- Select particles which behave like a MIP in the Electromagnetic Layers ( Eloss $_{1}<\mathrm{GeV}$ )

- In 2010, particles are required to have the maximum energy deposit of any layer be in a Hadronic Layer.

- Instead, in 20II, particles are required to deposit > $70 \%$ of their observed energy in a Hadronic Layer.

In-situ probe of Tile Calorimeter response

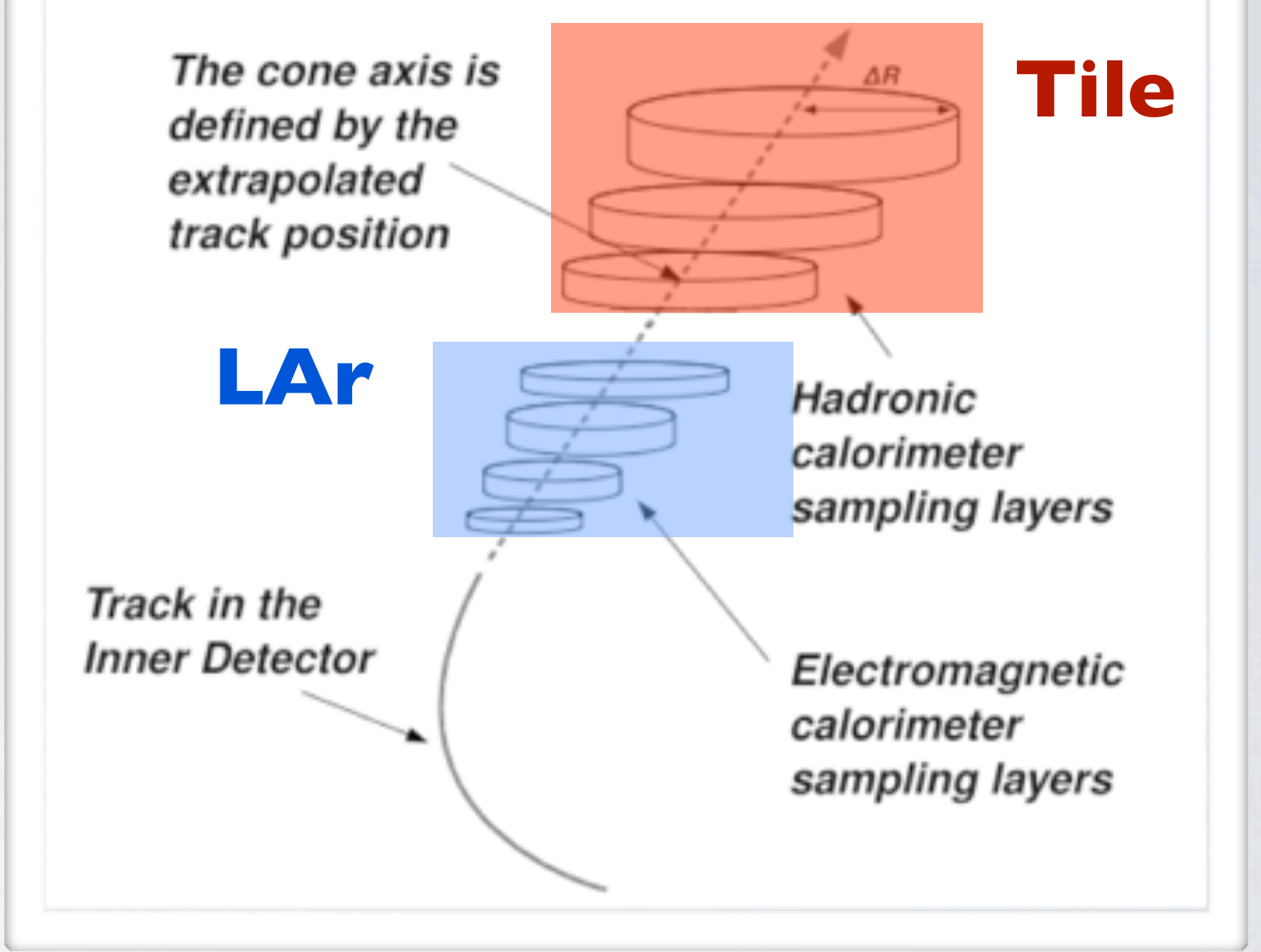




\section{Results}
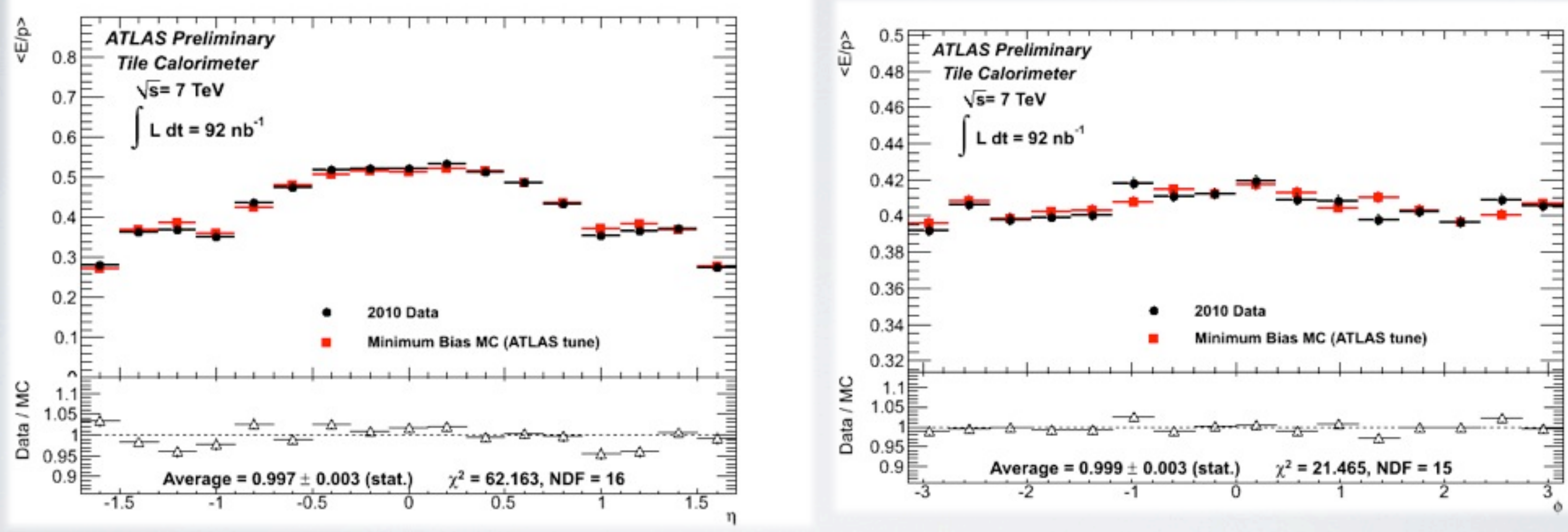

- Overall very good agreement between simulation and observation is measured, within 3\%

- Some small discrepancies in the region $0.8<|\eta|<1$. I, the transition between barrel and extended barrel ( $\sim 5 \%$ difference)

- 3 interesting areas stand out at $\phi=-1,1$ and $2.5(\sim 4 \%)$

In-situ probe of Tile Calorimeter response 


\section{Results}

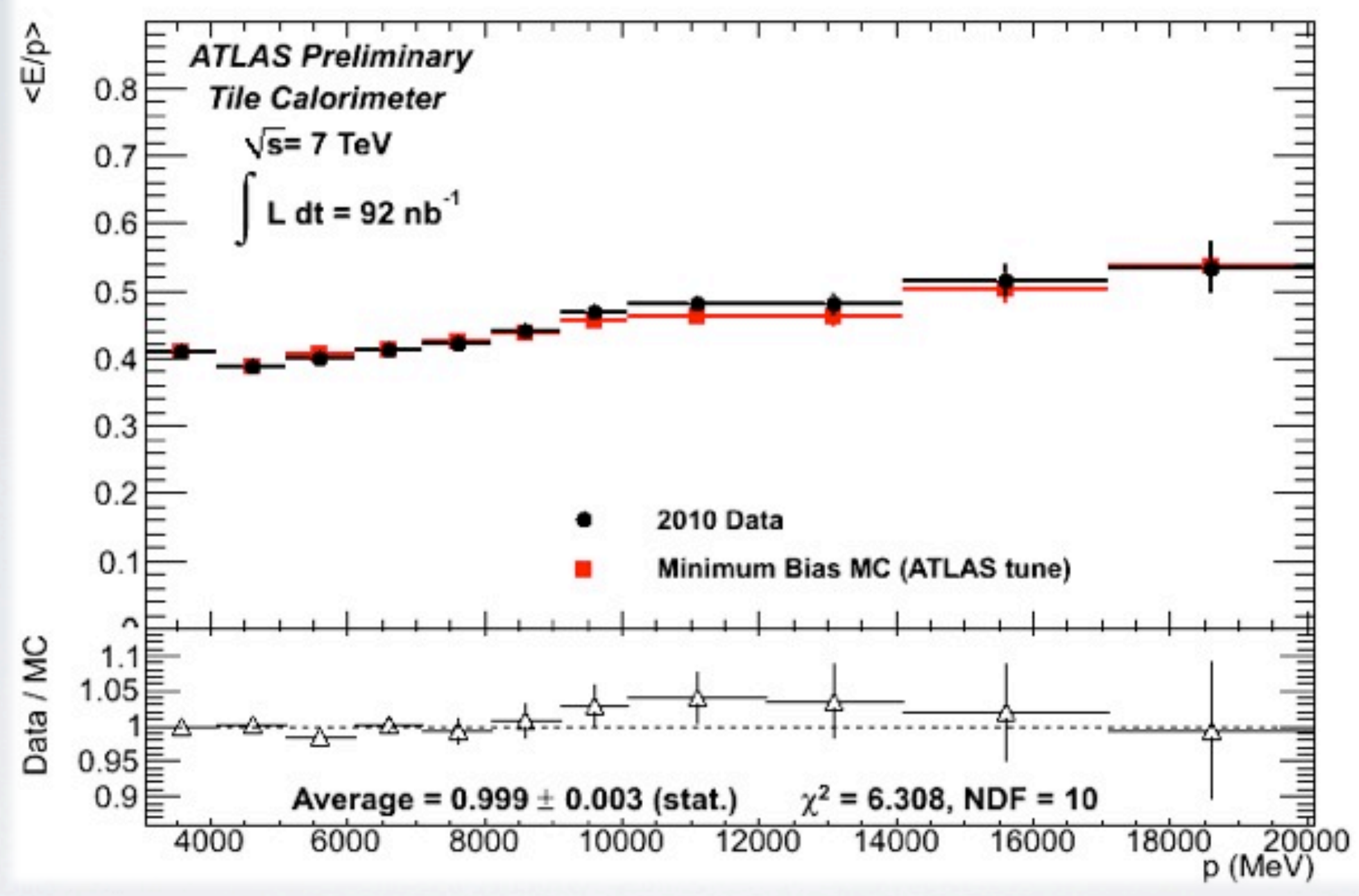

- Overall very good agreement between simulation and observation is measured, within $3 \%$

- A potentially interesting trend in the Data/MC ratio between 10 and $20 \mathrm{GeV}$

- Not enough particles are observed above $20 \mathrm{GeV}$ to measure the agreement

In-situ probe of Tile Calorimeter response 


\section{II Results}
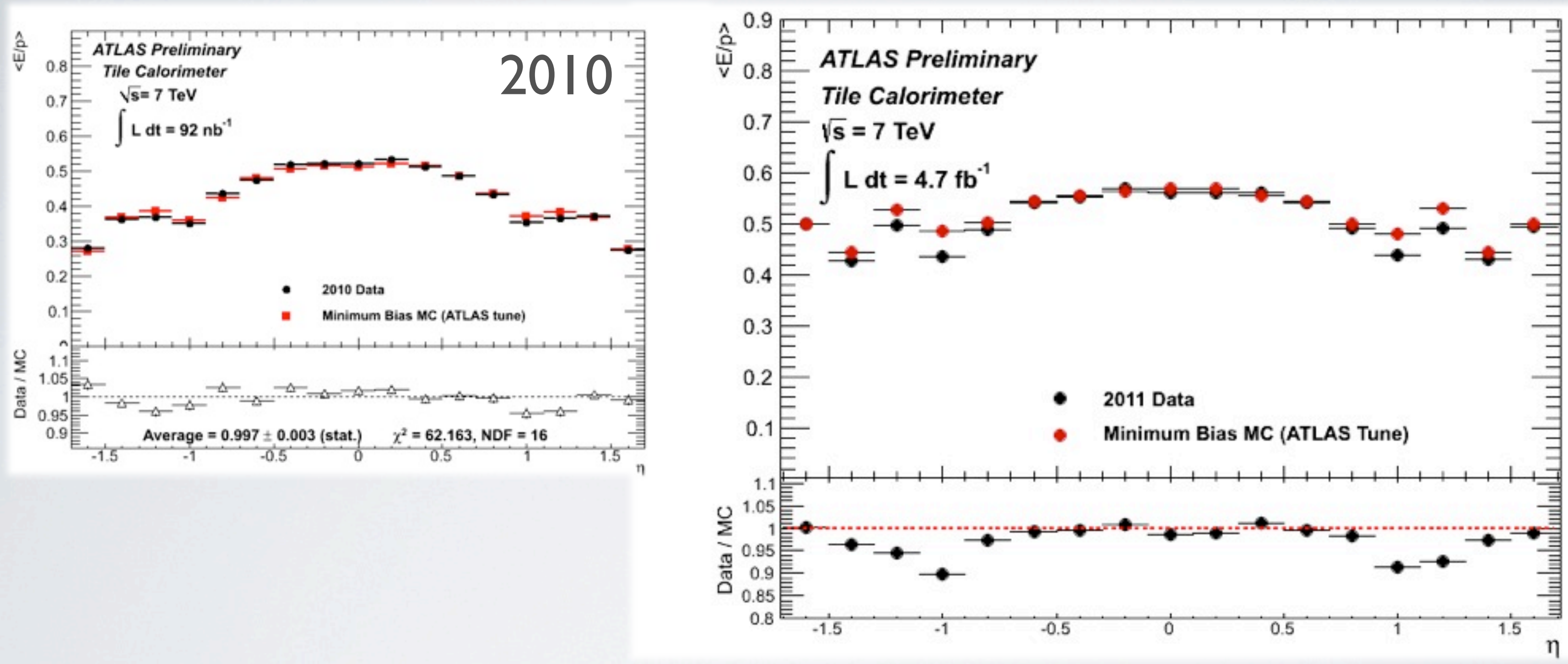

- $<E / p>$ has increased in the extended barrel with respect to $2010(\sim 0.4$ to $\sim 0.5)$

- Data/MC agreement has also decreased in this region ( $5 \%$ to 10\%)

- This increase in $\langle E / p>$ is attributable to the change in beam conditions. More pile-up and smaller bunch spacing leads to an increase in jets at higher values of $\eta$, which increase the baseline cell energy, even for isolated particles.

In-situ probe of Tile Calorimeter response 


\section{II Results}
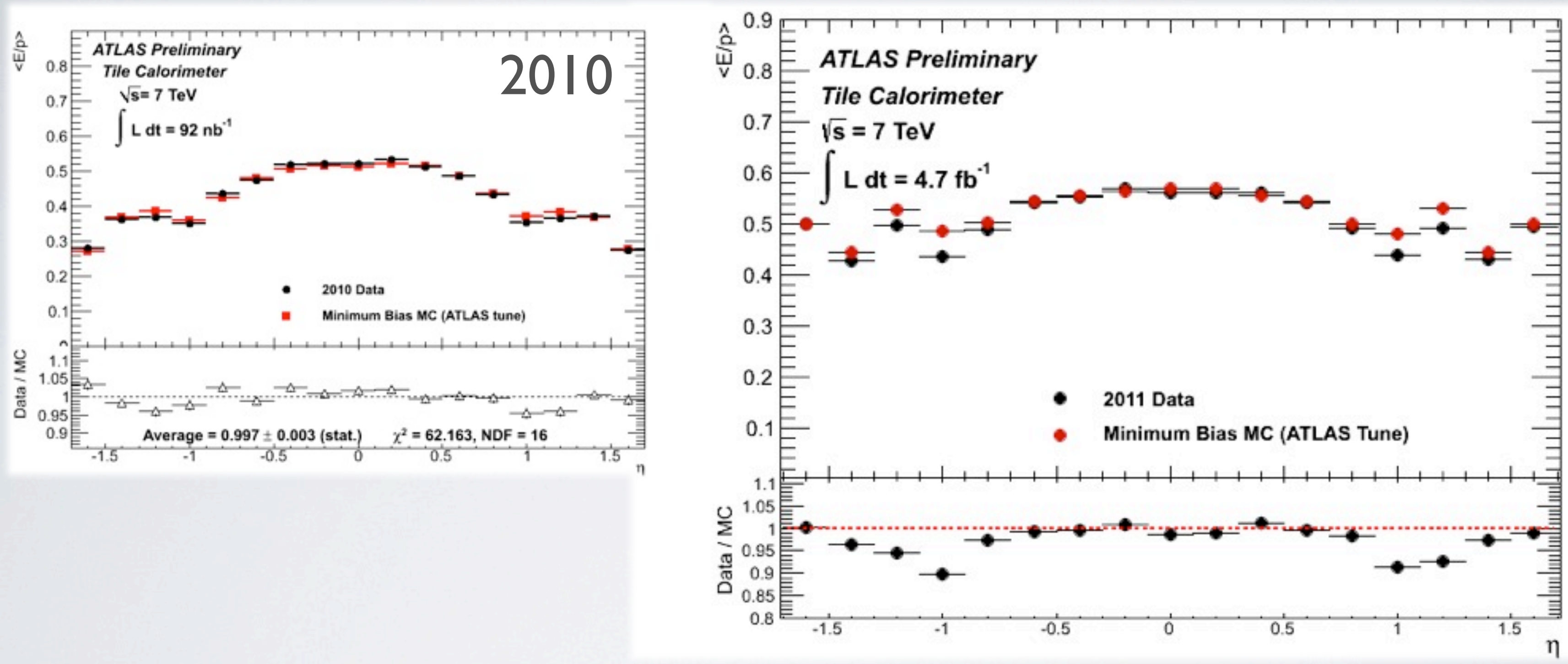

- Overall, $<E / p>$ has increased slightly more for the simulated sample.

- Simulated showers tend to be longer than observed showers.

- Thus particles in the simulation deposit more energy in Tile Calorimeter than those observed.

- This effect is amplified by the higher pileup conditions in 2011

In-situ probe of Tile Calorimeter response 


\section{I Results}
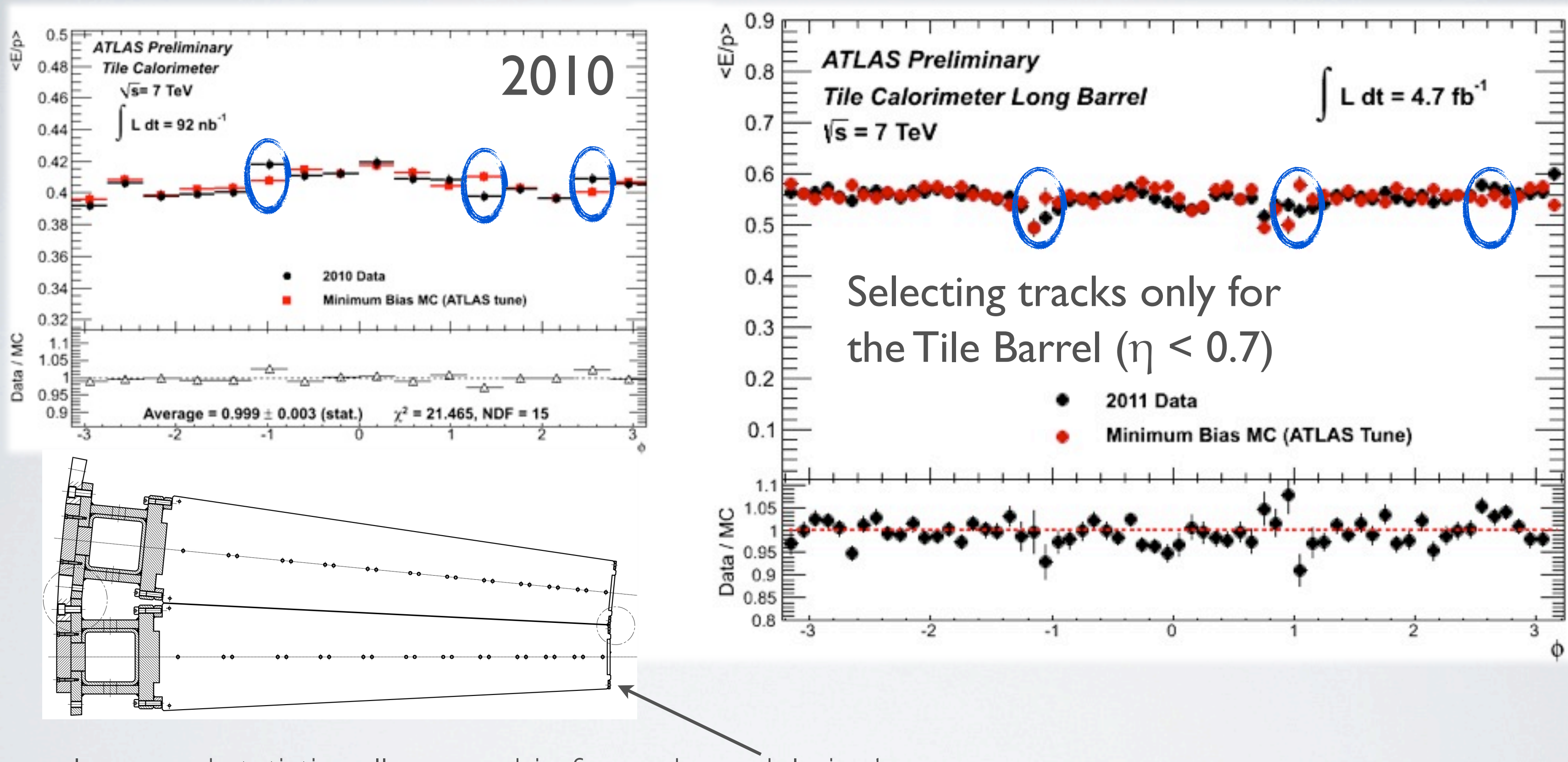

- Increased statistics allow one bin for each module in $\phi$

- Overall the good agreement between simulation and observation is similar to 2010

- The same areas of discrepancy can be identified, - I, I and 2.5 (this last is less significant)

In-situ probe of Tile Calorimeter response 


\section{I Results}
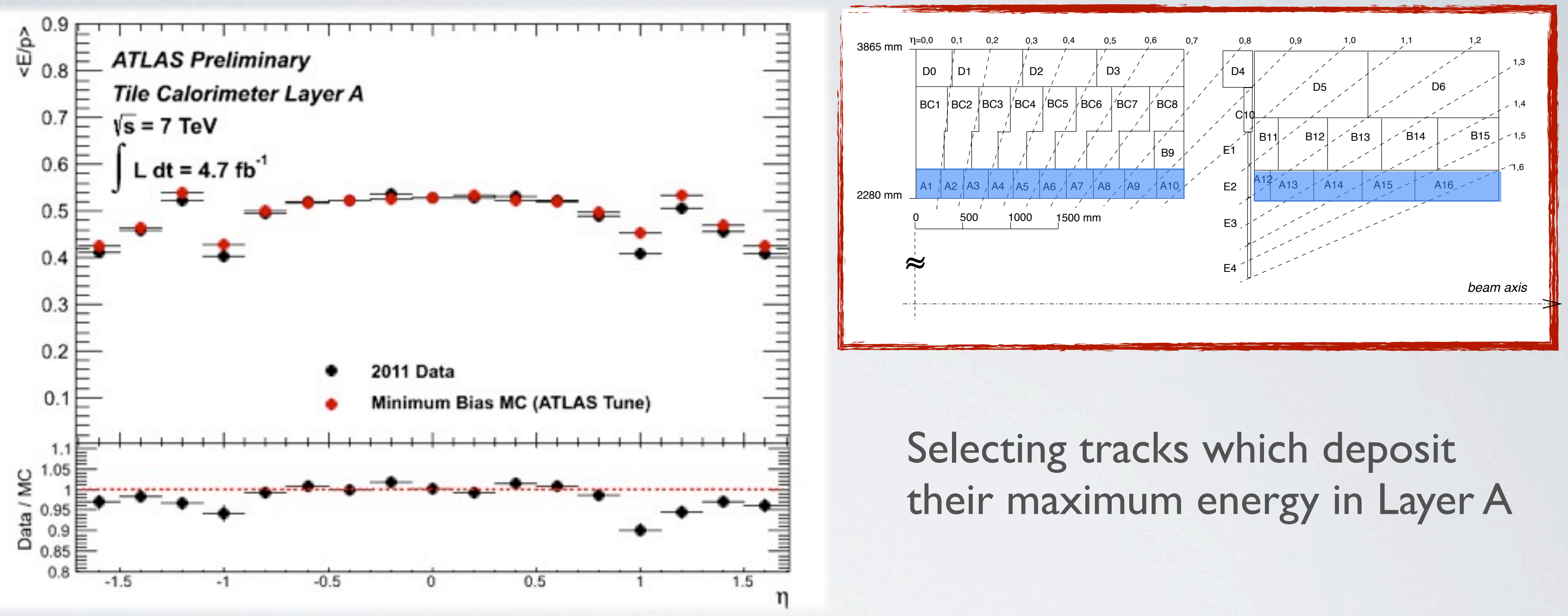

Selecting tracks which deposit their maximum energy in Layer $\mathrm{A}$

- Increased statistics also allow splitting into individual layers

- Focusing on Layer A shows even nicer agreement, particularly in the barrel

- Simulation is no longer consistently higher than observation

- By requiring the particle to shower early, the longer shower shape in simulation has less effect.

In-situ probe of Tile Calorimeter response 


\section{II Results}
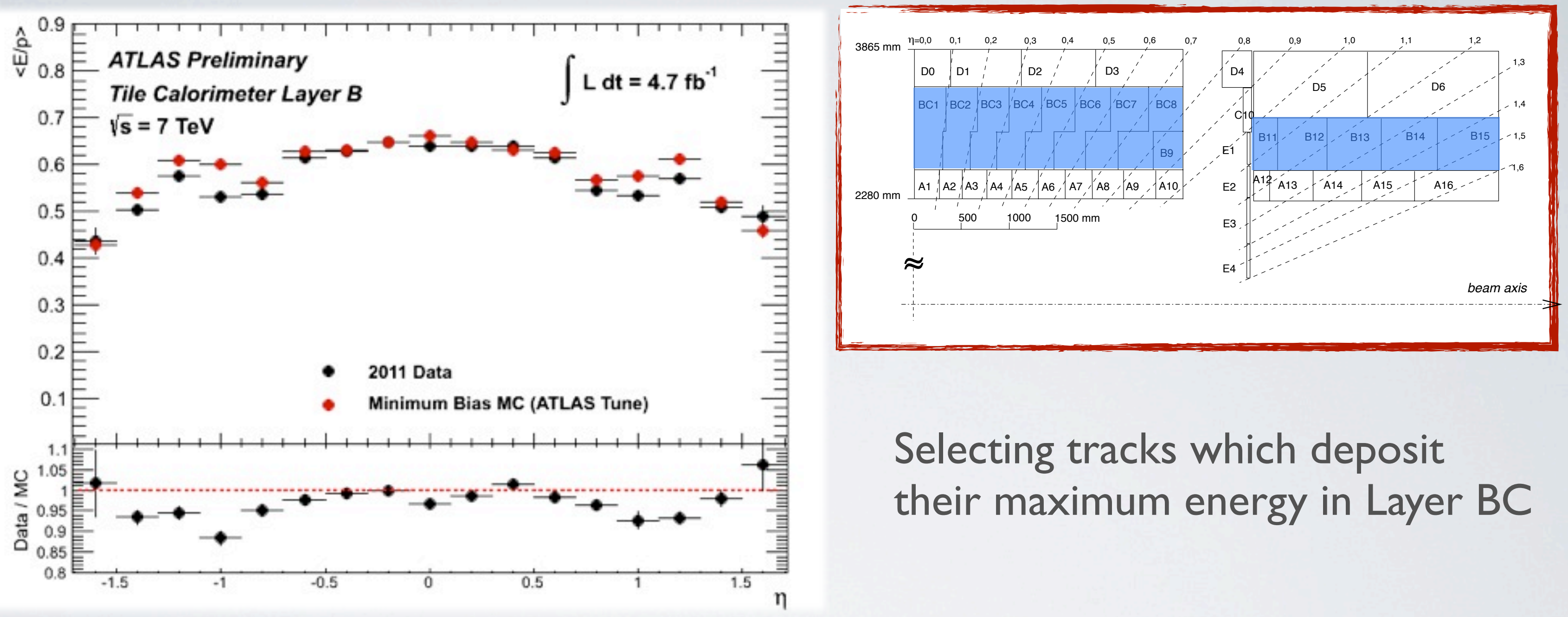

Selecting tracks which deposit their maximum energy in Layer BC

- Layer B has large discrepancies at $|\eta|=1$. Such particles barely graze the highlighted cells.

- $<E / p>$ is also higher than Layer A, for both simulation and observation.

- Particles which have a maximum deposit in Layer B have showers more contained within the hadronic calorimeter

In-situ probe of Tile Calorimeter response 


\section{Conclusions}

- This study is a firm validation of the energy scale of isolated hadrons.

- 2010 was proven to have good agreement due to calibration, and tuning simulation, with the test beam studies.

- A similar level of agreement to the combined calorimeter E/P analysis.

- 201 I shows very good agreement despite a large change in beam conditions.

- This is a success for the simulation of pile-up and the performance of energy reconstruction techniques.

- Studies such as these are used as feedback to improve the next iteration of simulation. 
BACKUP 


\section{MBTS}

- The Minimum Bias Trigger Scintillator (MBTS) is a set of 32 scintillating plates covering the range $2.1<|\eta|<3.8$.

- Fibers from these scintillating plates extend radially to photomultipliers in the TileCal girder

- The MBTS was used to trigger minimum bias events in early data taking.

\section{Beam Pipe}

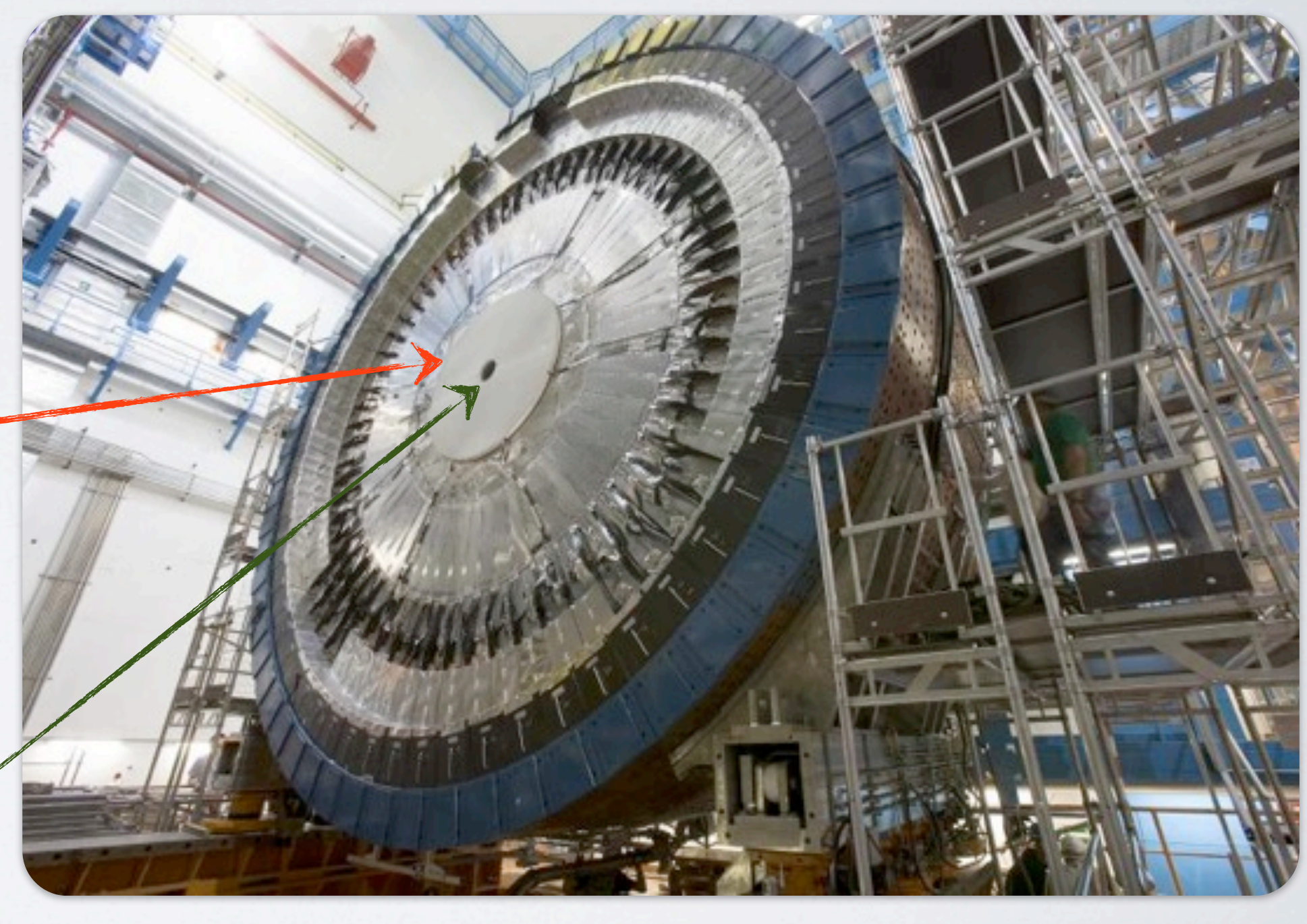

